medRxiv preprint doi: https://doi.org/10.1101/2020.10.01.20205450; this version posted October 4, 2020. The copyright holder for this preprint (which was not certified by peer review) is the author/funder, who has granted medRxiv a license to display the preprint in perpetuity. It is made available under a CC-BY-NC-ND 4.0 International license .

\title{
A multi-scale integrated analysis identifies KRT8 as a pan-cancer early biomarker
}

Madeleine K. D. Scott ${ }^{1,2,3}$, Michael G. Ozawa4, Pauline Chu, Maneesha Limaye ${ }^{5}$, Viswam S. Nair ${ }^{6,7,8}$, Steven Schaffert ${ }^{2,3}$, Albert C. Koong ${ }^{9}$, Robert West ${ }^{4}$, Purvesh Khatri ${ }^{2,3^{*}}$

\section{Biophysics Graduate Program, Stanford University, Stanford, CA, USA}

2. Center for Biomedical Informatics Research, Department of Medicine, Stanford University, Stanford, CA, USA

3. Institute for Immunity, Transplantation and Infection, Department of Medicine, Stanford University, Stanford, CA, USA

4. Department of Pathology, Stanford University, Stanford, CA, USA

5. Department of Pediatrics, Children's Hospital of Orange County, University of Irvine, California, Orange, CA, USA

6. Clinical Research Division, Fred Hutchinson Cancer Research Center, Seattle, WA, USA

7. Division of Pulmonary \& Critical Care Medicine, University of Washington, Seattle, WA, USA

8. Department of Radiology, Stanford University, Stanford, CA, USA

9. Department of Radiation Oncology, MD Anderson Cancer Center, Houston, TX, USA

*Correspondence should be addressed to P.K (pkhatri@stanford.edu), 2050 Biomedical Informatics Building, 240 Pasteur Dr, Stanford, CA 94305-5301

An early biomarker would transform our ability to screen and treat patients with cancer. The large amount of multi-scale molecular data in public repositories from various cancers provide unprecedented opportunities to find such a biomarker. However, despite identification of numerous molecular biomarkers using these public data, fewer than $1 \%$ have proven robust enough to translate into clinical practice ${ }^{1}$. One of the most important factors affecting the successful translation to clinical practice is lack of real-world patient population heterogeneity in the discovery process. Almost all biomarker studies analyze only a single cohort of patients with the same cancer using a single modality. Recent studies in other diseases have demonstrated the advantage of leveraging biological and technical heterogeneity across multiple independent cohorts to identify robust disease biomarkers. Here we analyzed 17149 samples from patients with one of 23 cancers that were profiled using either DNA methylation, bulk and single-cell gene expression, or protein expression in tumor and serum. First, we analyzed DNA methylation profiles of 9855 samples across 23 cancers from The Cancer Genome Atlas (TCGA). We then examined the gene expression profile of the most significantly hypomethylated gene, KRT8, in 6781 samples from 57 independent microarray datasets from NCBI GEO. KRT8 was significantly overexpressed across cancers except colon cancer (summary effect size $=1.05 ; \mathrm{p}<0.0001$ ). Further, single-cell RNAseq analysis of 7447 single cells from lung tumors showed that genes that significantly correlated with KRT8 ( $\mathrm{p}<$ 0.05) were involved in p53-related pathways. Immunohistochemistry in tumor biopsies from 294 patients with lung cancer showed that high protein expression of KRT8 is a prognostic marker of poor survival (HR $=1.73, \mathrm{p}$ $=0.01$ ). Finally, detectable KRT8 in serum as measured by ELISA distinguished patients with pancreatic cancer from healthy controls with an $A U R O C=0.94$. In summary, our analysis demonstrates that KRT8 is (1) differentially expressed in several cancers across all molecular modalities and (2) may be useful as a biomarker to identify patients that should be further tested for cancer.

\section{Introduction}

Most of the public health burden of cancer results from our inability to detect tumors before they become untreatable ${ }^{2}$. For instance, non-small cell lung cancer (NSCLC), the leading cause of cancer deaths worldwide, progresses from early to advanced stages over a year ${ }^{3}$. Early detection of NSCLC is shown to substantially NOTE: This preprint reports new research that has not been certified by peer review and should not be used to guide clinical practice. 
improve survival through surgical resection of the tumor"; however, after the cancer has metastasized, surgical intervention does not improve patient outcomes 5 . This critical need for early cancer biomarkers motivated the creation of consortiums like the TCGA ${ }^{6}$. Since the first TCGA data was released in 2006, there have been hundreds of putative molecular biomarkers proposed across all cancer types, with most focusing on gene expression biomarkers ${ }^{7,8}$. However, most gene signature biomarkers were identified in only one cancer type or subtype, and very few ever proved to be viable for clinical use ${ }^{8,9}$. Many proposed signatures failed to translate into clinical practice because they could not be replicated in outside cohorts or performed poorly when clinical data was considered ${ }^{10}$.

DNA methylation profiles have been shown to carry additional information to either genomic or expression data ${ }^{11,12}$. Yang et al. demonstrated that TCGA methylation data could identify clinically relevant subsets of patients with breast cancer that could not be classified by gene expression ${ }^{13}$. Others have documented the prognostic ability of other epigenetic signatures in colon, lung, and pancreatic cancer ${ }^{14-16}$.

However, the bulk of putative methylation biomarkers are limited to a single disease and face the same clinical translation issues as gene expression biomarkers ${ }^{17}$. To increase the probability that a methylation biomarker is useful in clinical practice, it is critical to demonstrate a robust functional and translational relevance of the differentially methylated genes in multiple cohorts ${ }^{18}$. Additionally, the focus on single-cancer biomarkers has raised concerns about the potential to overlook common epigenetic drivers of cancer ${ }^{19}$.

In this study, we performed a pan-cancer analysis of TCGA DNA methylation data from 9855 tissue samples across 23 cancers to inform subsequent gene expression, proteomic, and clinical outcome analyses. The methylation samples were divided into discovery (2019 samples across 10 cancers) and validation (7836 samples across 21 cancers). KRT8 was the most significant differentially methylated gene across cancers. We next examined the gene expression profile of KRT8 in 6781 samples from 57 independent microarray datasets in five solid tumor cancers (breast, colon, pancreatic, ovarian and lung) from NCBI GEO ${ }^{20}$, and found KRT8 to be universally overexpressed. Our analysis of intra-cellular gene-KRT8 expression correlations in 7447 single cells derived from lung tumor biopsies found KRT8 is correlated with genes involved in p53-related pathways. We validated these correlations in gene expression microarrays of 1276 tissue biopsies from patients with lung cancer. We examined the prognostic relevance of tumor KRT8 protein in 294 tissue microarrays (TMAs) from patients with lung cancer. We then calculated the prognostic value of tumor KRT8 gene expression in pancreatic

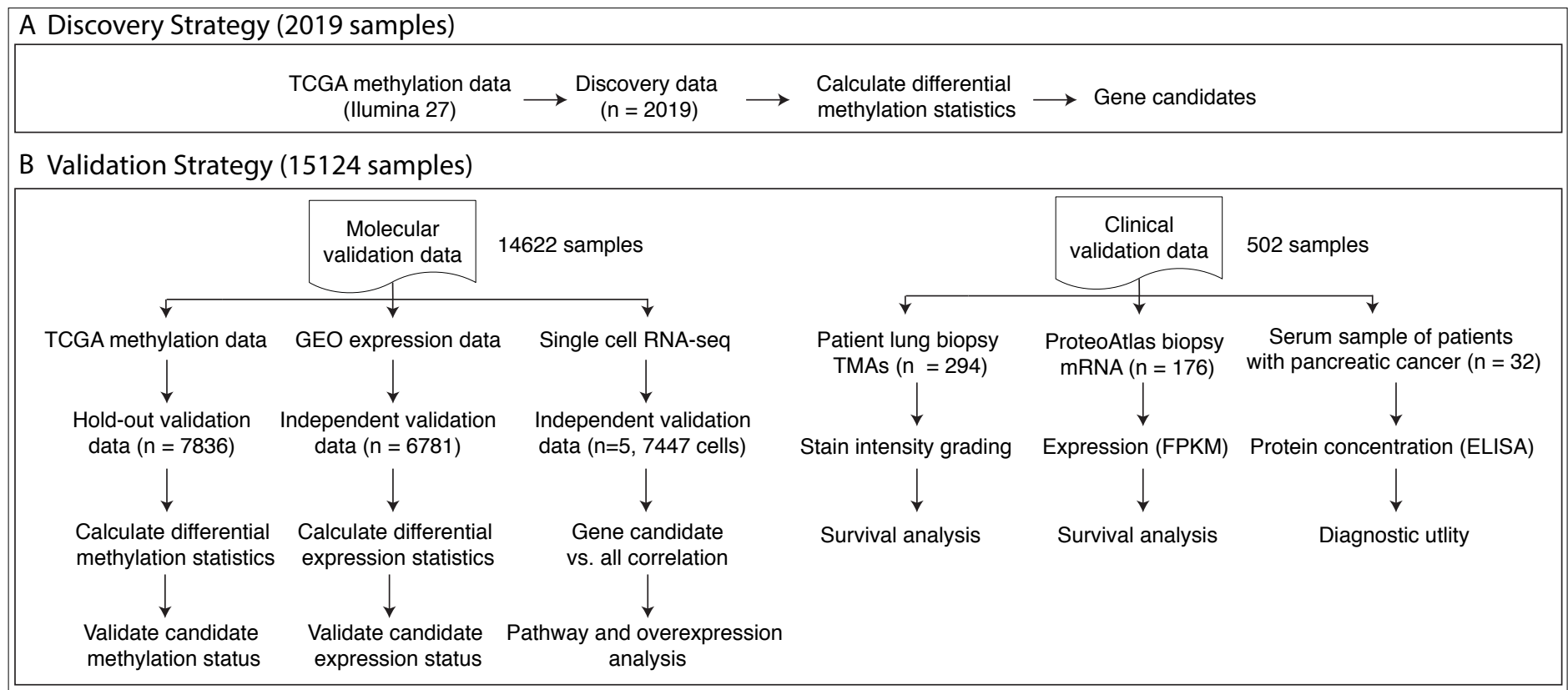

Figure 1. Analysis overview. (A) DNA methylation data from TCGA for 10 cancers comprising 2019 samples profiled using the Illumina 27 platform were used for discovery. (B) Validation data comprised of 15,124 samples profiled using either DNA methylation, bulk and single cell gene expression, or tumor and serum protein. 
cancer with data from Protein Atlas. Finally, we validated the potential of KRT8 as non-invasive biomarker with serum KRT8 in 32 pancreatic patients and 6 healthy controls from Stanford Hospital. An overview of this analysis is displayed in Figure 1.

\section{Methods}

\section{Data Collection from Public Repositories - TCGA and GEO}

All methylation and transcriptome data used in our analyses are publicly available. We downloaded all available DNA methylation data the TCGA data portal (https://tcga-data.nci.nih.gov/tcga/tcgaHome2.jsp) irrespective of cancer on May 19, 2018. We excluded data for cancers where less than two non-cancerous samples were profiled, which resulted in DNA methylation data for 9855 samples across 23 cancers. For DNA methylation profiling, samples from these 23 cancers were profiled using either the Infinium HM27 array (27,578 CpG site targeting probes) or Infinium HM450 array (485,577 CpG site targeting probes). All data was generated and processed by The Cancer Genome Atlas research network as described previously ${ }^{6,19}$. We used data profiled on the HM27 array as our discovery cohort (10 cancers, 2019 samples) and data profiled on the HM450 array (21 cancers, 7836 samples) as validation.

For gene expression, we downloaded whole transcriptome data for 6,781 tumor biopsies across 57 independent datasets profiled using microarrays from the NCBI GEO. All datasets were required to measure gene expression in a minimum of two non-cancerous tissue samples. These tumor biopsies came from a patient with breast, lung, pancreatic, ovarian or colon cancer.

\section{Data Processing and Effect Size Estimation}

We ensured all downloaded gene expression data was log2-transformed. For each gene, we calculated change in expression in a tumor biopsy as Hedges' $g$ with adjustment for small sample size because it captures both the fold change and variance. We have previously used Hedges' $g$ to generate robust gene signatures with diagnostic and prognostic value ${ }^{21,22}$. We used the random-effects inverse variance meta-analysis using Dersimonian-Laird method to calculate a summary effect size (ES) across datasets for each gene ${ }^{23}$. We chose Dersimonian-Laird as our previous work has shown it to be a good compromise between more conservative meta-analysis methods (Sidik-Jonkman, Hedges-Olkin, empiric Bayes, restricted maximum likelihood) and lenient methods (HunterSchmidt ${ }^{23}$. If multiple probes mapped to a gene, the effect size for each gene was summarized via the fixed effect inverse-variance model. We corrected p-values for summary effect-sizes for multiple hypotheses testing using Benjamini-Hochberg false discovery rate (FDR) correction. ${ }^{24}$ We removed one cancer at a time and applied both meta-analysis methods at each iteration to avoid influence of a specific cancer with a large sample size on the results.

\section{Survival Analysis and Modeling}

We used a right-censored model to fit survival data with the survival package in the $\mathrm{R}$ statistical computing environment (Version 3.5.1). We fit univariate and multivariate Cox proportional hazards models onto survival data using the coxph function. We confirmed the proportional hazard assumption with the cox.zph function.

\section{Human Plasma Samples}

Our study includes 32 human EDTA blood plasma samples collected between January 2007 and October 2011 from identically staged patients with advanced pancreatic ductal adenocarcinoma treated at Stanford University Medical Center under an institutional review board-approved protocol. All plasma samples were collected from untreated (de novo) patients with biopsy- proven pancreatic adenocarcinomas. Median age at blood collection was 68 years (range 37-84 years). All patients were treated with gemcitabine-based chemotherapy and the majority also received radiotherapy. As a control group, 6 additional plasma samples were collected from agematched, healthy volunteers under an IRB-approved protocol. Immediately after acquisition, blood samples were centrifuged and aliquots of plasma stored at $-80^{\circ} \mathrm{C}$. 
medRxiv preprint doi: https://doi.org/10.1101/2020.10.01.20205450; this version posted October 4, 2020. The copyright holder for this preprint (which was not certified by peer review) is the author/funder, who has granted medRxiv a license to display the preprint in perpetuity. It is made available under a CC-BY-NC-ND 4.0 International license .

\section{Enzyme-linked immunosorbent assay (ELISA)}

The serum biomarker concentration was measured with a commercially available human protein sandwich enzyme immunoassay kit with two mouse monoclonal antihuman antibodies (R\&D Systems, Inc., Minneapolis, MN, USA). All serum samples from patients and standards were incubated in microplate wells coated with the first mouse monoclonal anti-human biomarker antibody. After washing, a second antihuman biomarker antibody labeled with peroxidase (HRP) was added for subsequent incubation. The reaction between HRP and substrate (hydrogen peroxide and tetramethylbenzidine) resulted in color development and the intensities were measured with a microplate reader at an absorbance of $450 \mathrm{~nm}$. Concentrations of serum biomarkers were determined against a standard curve.

\section{Single cell data collection and processing}

We downloaded count matrices of 52,698 single cells from the tumor microenvironment of five lung cancer patient samples from Array Express (E-MTAB-6149) ${ }^{25}$. Of the total 52,698 cells, 7,447 originated from the tumor. We calculated the Pearson correlation between expression of KRT8 and all other measured genes within each tumor cell. For each KRT8-gene correlation, we required non-zero expression of both genes in a minimum of 25 cells. We removed correlations with a $\mathrm{p}$-value $\geq 0.05$.

\section{KRT8 Expression in Patients with Pancreatic Cancer from The Protein Atlas}

We downloaded prognostic information for 176 pancreatic cancer patients stratified by tumor KRT8 expression from The Protein Atlas ${ }^{26} \quad$ (https://www.proteinatlas.org/ENSG00000170421KRT8/pathology/tissue/pancreatic+cancer). We stratified patients based on median KRT8 expression of the cohort. Patient samples originated from the TCGA data repository. All counts are reported as Fragments Per Kilobase of exon per Million reads (FPKM).

\section{TMA cohort, and immunohistochemistry}

Patient samples were retrieved from the surgical pathology archives at the Stanford Department of Pathology and linked to a clinical database using the Cancer Center Database and STRIDE Database tools from Stanford. Patients who had surgically treated disease and paraffin embedded samples from 1995 through June, 2010 were included. Surgical specimens that contained viable tumor from slides were reviewed by a board-certified pathologist (RBW) to build the Stanford Lung Cancer TMA as described previously. The area of highest tumor content was marked for coring blocks corresponding to the slides using $0.6 \mathrm{~mm}$ cores in duplicate arrays as previously described ${ }^{27}$. These cores were aligned by histology and stage and negative controls included a variety of benign and malignant tissues that included normal non-lung tissue, abnormal non-lung tissue, placental markers, and normal lung ${ }^{27}$. Normal lung consisted of a specimen adjacent, but distinct, from tumor over the years 1995 through 2010 to assess the variability of staining by year. OligoDT analysis was performed on the finished array to assess the architecture of selected cores and adequacy of tissue content prior to target immunohistochemistry (IHC) analysis. Serial $4 \mu \mathrm{m}$ sections were cut from FFPE specimens and processed for IHC using the Ventana BenchMark XT automated immunostaining platform (Ventana Medical Systems/Roche, Tucson, AZ). Rabbit monoclonal anti-Cytokeratin 8 (phospho S431) antibody was obtained from Abcam (ab109452, Burlingame, CA). Mouse monocolonal Anti-Cytokeratin 8 antibody was also obtained from Abcam (ab9023, Burlingame, CA). The intensity of KRT8 immunostaining was graded from 1-4 as determined by an independent pathologist who was blinded to patient outcome.

\section{Results}

\section{Integrated analysis of TCGA methylation data identifies KRT8 as hypomethylated across cancers}

We identified 23 cancers that had methylation data and at least two healthy controls per cancer from TCGA. We split the resulting 9855 samples into discovery cohorts (2019 samples from 10 cancers profiled using the Illumina 
27 platform) and the validation cohorts (7836 samples from 21 cancers profiled using the Illumina 450 platform) for validation. In order to avoid the potential influence of a single cancer on the results due to unequal sample sizes or other unknown confounding factors among cohorts, we performed a "leave-one-cancer-out" analysis. We hypothesized that the resulting set of methylation sites, irrespective of the set of cancers analyzed, would constitute a robust methylation signature across cancers. We identified 1,801 differentially methylated genes (1,081 hyper- and 720 hypomethylated, FDR < 5\%) across all cancers (Figure 1A and Supplementary Figure 1A). We did not remove differentially methylated sites with significant heterogeneity for two reasons. First, heterogeneity is expected due to known heterogeneity within and between cancers. Second, we have previously shown that when combining across multiple datasets, filtering by heterogeneity removes higher proportion of true positives than false positives ${ }^{23}$. In the validation cohorts, which used Illumina 450 platform, we found 1083 out of 1,801 sites were differentially methylated across all cancers (FDR < 5\%; Figure 1B and Supplementary Figure 1B).

Our discovery analysis found several previously reported differentially methylated genes. The hypomethylated genes across all cancers in the discovery cohort included $C L D N 4^{28}$ (discovery ES $=-1.86, \mathrm{p}=8.0 \mathrm{e}-7$; validation ES $=-0.56, \mathrm{p}=1.55 \mathrm{e}-06$ ) and $S F N^{29}$ (discovery $E S=-0.96, \mathrm{p}=2.01 \mathrm{e}-7$; validation $E S=-0.94, \mathrm{p}=9.4 \mathrm{e}-10$ ) that have been

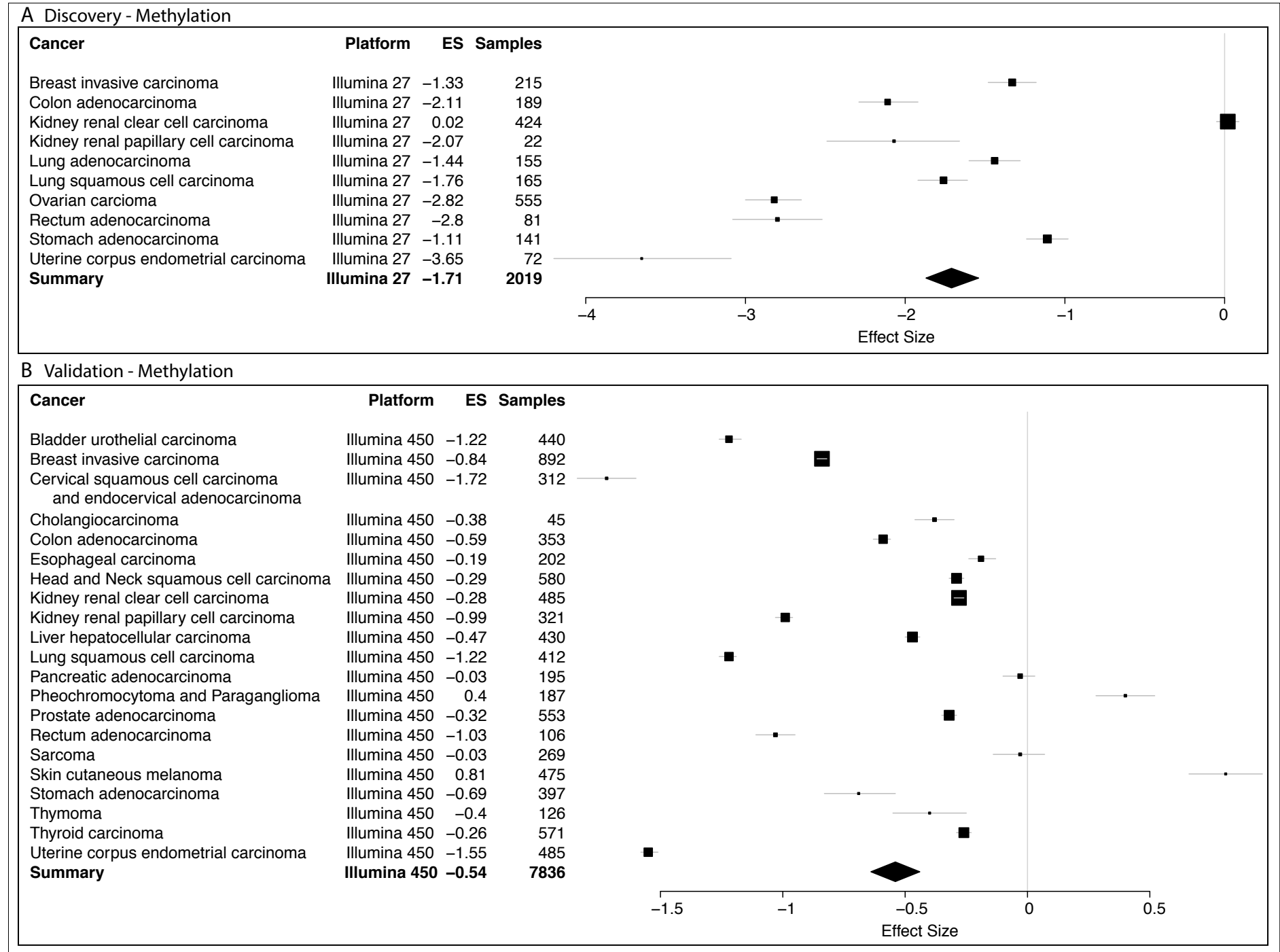

Figure 2. KRT8 is hypomethylated across 23 cancers. Differential DNA methylation of KRT8 in (A) discovery (10 cancers, 2019 samples, Illumina 27 platform) and (B) validation (21 cancers, 7836 samples, Illumina 450 platform) tumor biopsy samples compared to control non-cancerous tissue. X axes represent standardized mean difference in DNA methylation between cancer and control samples, computed as Hedges' $g$, in $\log _{2}$ scale. The size of a rectangle is inversely proportional to the SEM in the corresponding cancer cohort. Whiskers represent the $95 \%$ confidence interval. A diamond represents a summary effect size for KRT8 across cancers. Width of a diamond represents the $95 \%$ confidence interval of summary effect size. SEM: standard error of mean. 


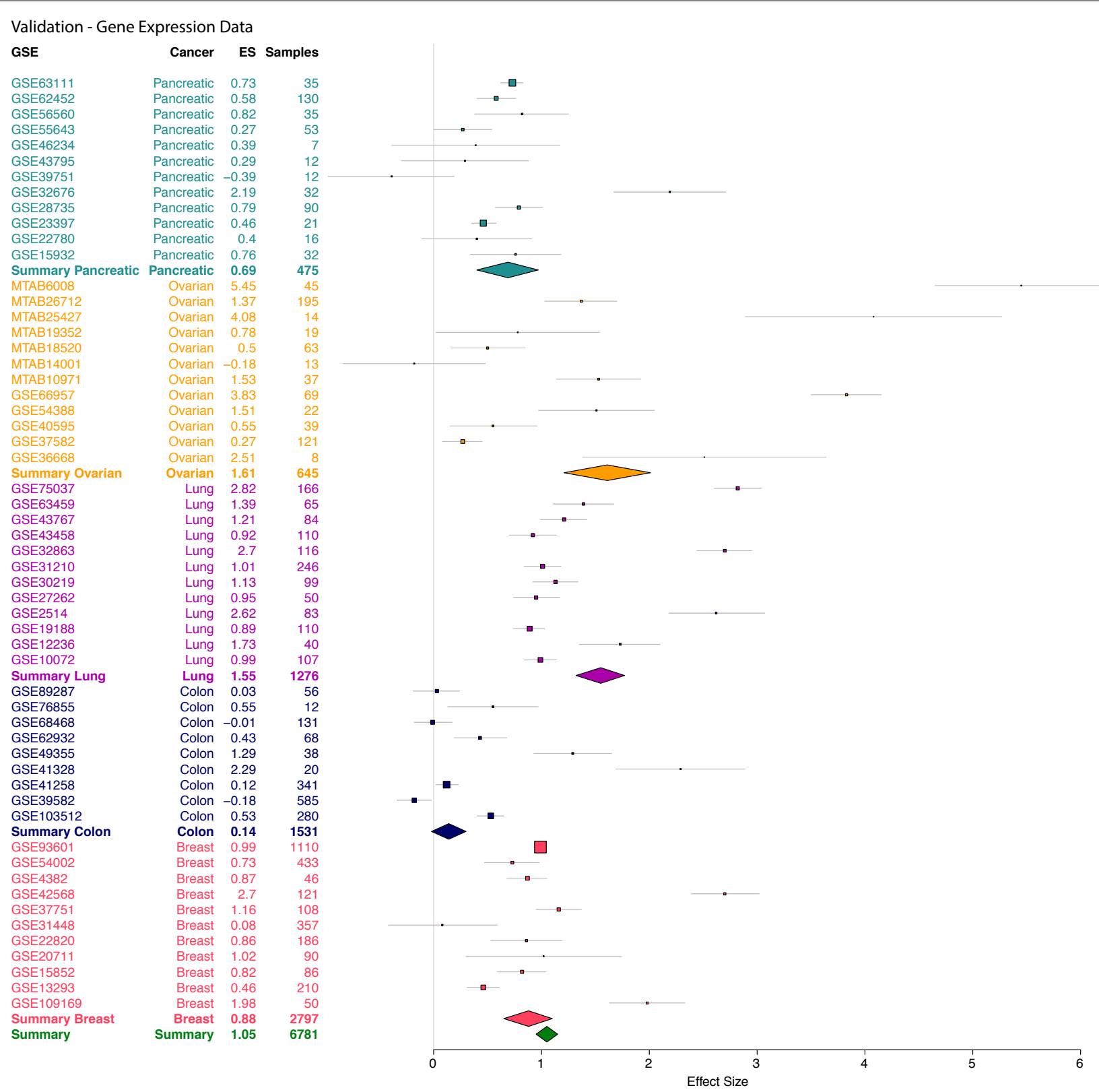

Figure 3. KRT8 gene is overexpressed in five cancers. The $x$ axes represent standardized mean difference in gene expression between cancer and control samples, computed as Hedges' $\mathrm{g}$, in $\log _{2}$ scale. The size of a rectangle is inversely proportional to the SEM in the cohort. Whiskers represent the 95\% confidence interval. A diamond represents a summary effect size for KRT8 across cancers. Green diamond represents summary effect size across all cancers. Width of a diamond represents the $95 \%$ confidence interval of summary effect size. SEM: standard error of mean.

previously shown to promote cancer cell proliferation (Ehrlich 2009), whereas the hypermethylated genes included known tumor suppressors such as $S O X 1^{30}$ (discovery ES = 1.05, $\mathrm{p}=3.4 \mathrm{e}-08$; validation ES = 1.08, $\mathrm{p}=$ 4.4e-22), TWIST ${ }^{31}$ (discovery ES = 0.89, $\mathrm{p}=1.5 \mathrm{e}-5$; validation ES = 0.59, $\mathrm{p}=4.3 \mathrm{e}-16$ ), and GATA4 ${ }^{32}$ (discovery ES = $0.92, \mathrm{p}=1.7 \mathrm{e}-6$; validation $\mathrm{ES}=0.38, \mathrm{p}=3.77 \mathrm{e}-12)$. KRT8 was the most statistically significant hypomethylated gene after multiple hypothesis correction (discovery ES =-1.71, p = 3.2e-7, FDR=9.15e-6; Figure 2A), but was unchanged in renal clear cell carcinoma. KRT8 was also hypomethylated in the validation cohorts across all cancers except pheochromatoma/paraganglioma and melanoma (validation ES=-0.69, p = 3.3e-15, FDR = 4.0e-14; Figure 2B) (Figure 2). 


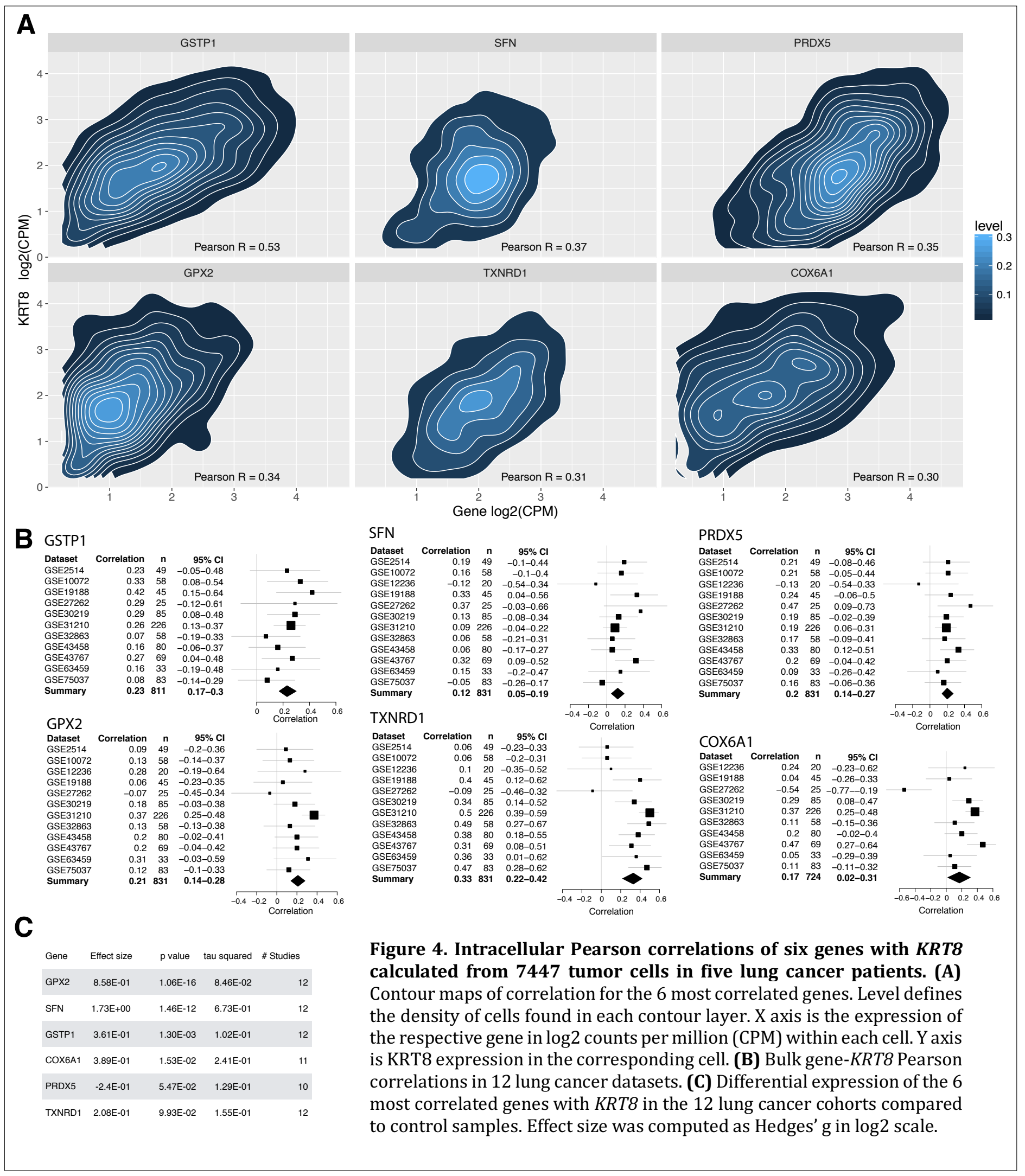

\section{Multi-cohort gene expression analysis demonstrates KRT8 is over-expressed in five cancers}

Hypomethylation and hypermethylation typically lead to over- and under-expression of the corresponding gene, respectively. ${ }^{33}$ Therefore, we hypothesized that hypo- or hyper-methylated genes across multiple cancers will be over- or under-expressed across multiple cancer compared to control samples. Arguably, we could use gene expression data for the same samples from TCGA. However, we decided to use gene expression data from 
completely independent cohorts from a different source to increase stringency of our analysis. Therefore, to test

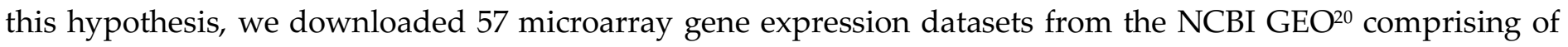
6781 samples (4870 cases, 1911 controls) obtained from human tissue biopsies of five cancers: breast, colon, lung adenocarcinoma, ovarian, or pancreatic. These 57 datasets included broad biological and technical heterogeneity, such as treatment protocols, demographics, collection year, and microarray platforms to further increase the stringency of our analysis and identify robust signals that persist despite these potential sources of vznoise.

Differential gene expression meta-analysis across all 6781 samples identified overexpression of known oncogenes such as ERBB2 (ES =0.51, $\mathrm{p}=6.22 \mathrm{e}-13), K R A S(\mathrm{ES}=0.43, \mathrm{p}=2.90 \mathrm{e}-9), C C N D 1(\mathrm{ES}=0.25, \mathrm{p}=7.34 \mathrm{e}-3)$, and VEGFA (ES $=0.42, \mathrm{p}=2.19 \mathrm{e}-06)$. Housekeeping genes did not show a change in expression between control and cancer, such as B2M (ES=0.12, p=.25), HBS1L $(E S=-0.08, \mathrm{p}=0.15)$, or EMC7 $(\mathrm{ES}=0.18, \mathrm{p}=0.09)^{34,35}$.

Next, we calculated the Spearman correlation between the discovery methylation ES and gene expression ES in the 1,801 differentially methylated genes as $-0.21(\mathrm{p}=1.27 \mathrm{e}-19)$, which in line with previous studies ${ }^{36}$ that $^{2}$ examined intra-sample methylation-expression correlation (Supplementary Figure 2).

Finally, we found that hypomethylation of KRT8 led to overexpression in multiple cancers compared to healthy samples (ES=1.05, p=2.8e-27, FDR=2.0e-24; Figure 3). KRT8 was over-expressed in pancreatic cancer (ES=0.69, $\mathrm{p}=4.02 \mathrm{e}-08)$, ovarian cancer $(\mathrm{ES}=1.61, \mathrm{p}=1.93 \mathrm{e}-03)$, lung cancer $(\mathrm{ES}=1.55, \mathrm{p}=1.95 \mathrm{e}-13)$, and breast cancer $(\mathrm{ES}=0.88$, $\mathrm{p}=7.82 \mathrm{e}-10)$, but not in colon cancer $(\mathrm{ES}=0.14, \mathrm{p}=0.38)$.

\section{KRT8 overexpression is associated with a chemotherapy-resistant phenotype in vitro}

Chemotherapy resistance is responsible for more than $80 \%$ of cancer-related mortality. We investigated whether increased KRT8 expression is associated with chemotherapy resistance. We downloaded 100 samples in seven datasets from NCBI GEO across six cancers that contained both chemotherapy-resistant and chemotherapysensitive cell lines. KRT8 was consistently overexpressed across all chemo-resistant cancer cell lines (summary effect size $=0.76, \mathrm{p}=0.035$; Supplementary Figure 3). This result demonstrates a consistent association between KRT8 expression and chemotherapy resistance in vitro.

\section{Single cell analysis of KRT8 expression}

Single cell gene expression data has allowed researchers to probe intra-cellular gene-gene correlations, which in turn suggest gene interactions or a common regulator. We analyzed intra-cellular correlations between every gene and KRT8 with single cell RNA sequencing data of 7447 cells from tumor biopsies of five lung cancer patients. To calculate intra-cell gene-gene correlations, we correlated the expression of each gene to KRT8 expression in every cell. Several other keratin genes were positively correlated with KRT8. For example, KRT18 and KRT7 had Pearson correlation of 0.59 and 0.55, respectively, with KRT8. Next, we preformed pathway analysis of the 100 most positively and negatively correlated genes with KRT8 using the Reactome Knowledge Database $^{37}$. Thirty out of the 100 genes were not annotated in the Reactome Knowledge Database. We identified six significantly enriched pathways, each of which has been previously implicated in cancer progression (Figure 4A). The top three significantly enriched pathways were comprised of six unique genes: GSTP1, PRDX5, GPX2, TXNRD1, SFN, COX6A1 (Supplementary Table 1). Each of these six genes had an intra-cellular correlation with KRT8 expression $\geq 0.30$ (Figure 4A). All genes except GSTP1 are annotated in Reactome as involved in p53 signal transduction (Supplementary Table 1). However, GSTP1 is known to be a direct transcriptional target of p5338, further supporting the association between KRT8 and genes involved in the p53 pathway.

We next examined the correlation between the six genes and KRT8 in bulk lung adenocarcinoma gene expression data from microarrays of 1276 lung biopsy samples from 12 datasets. All genes were significantly correlated with KRT8 at sample level (Figure 4B). All genes except PRDX5 were overexpressed in lung adenocarcinoma compared to healthy patients (Figure 4C). The majority of these six genes were additionally overexpressed 
medRxiv preprint doi: https://doi.org/10.1101/2020.10.01.20205450; this version posted October 4, 2020. The copyright holder for this preprint (which was not certified by peer review) is the author/funder, who has granted medRxiv a license to display the preprint in perpetuity. It is made available under a CC-BY-NC-ND 4.0 International license .

across 5505 microarray samples from four cancers (breast, colon, ovarian, and pancreatic; Supplementary Table 2).

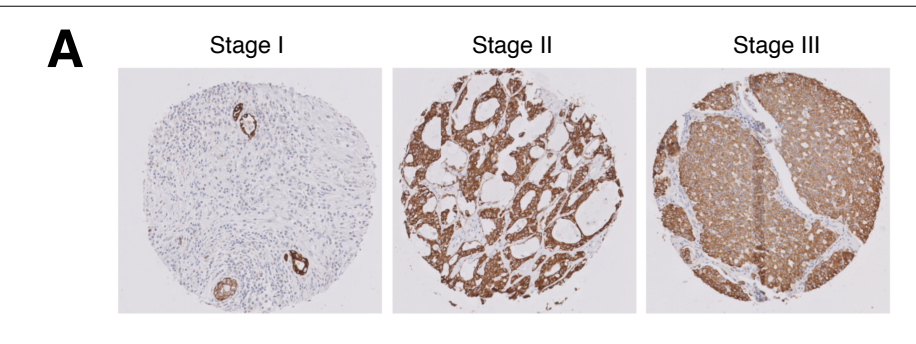

B
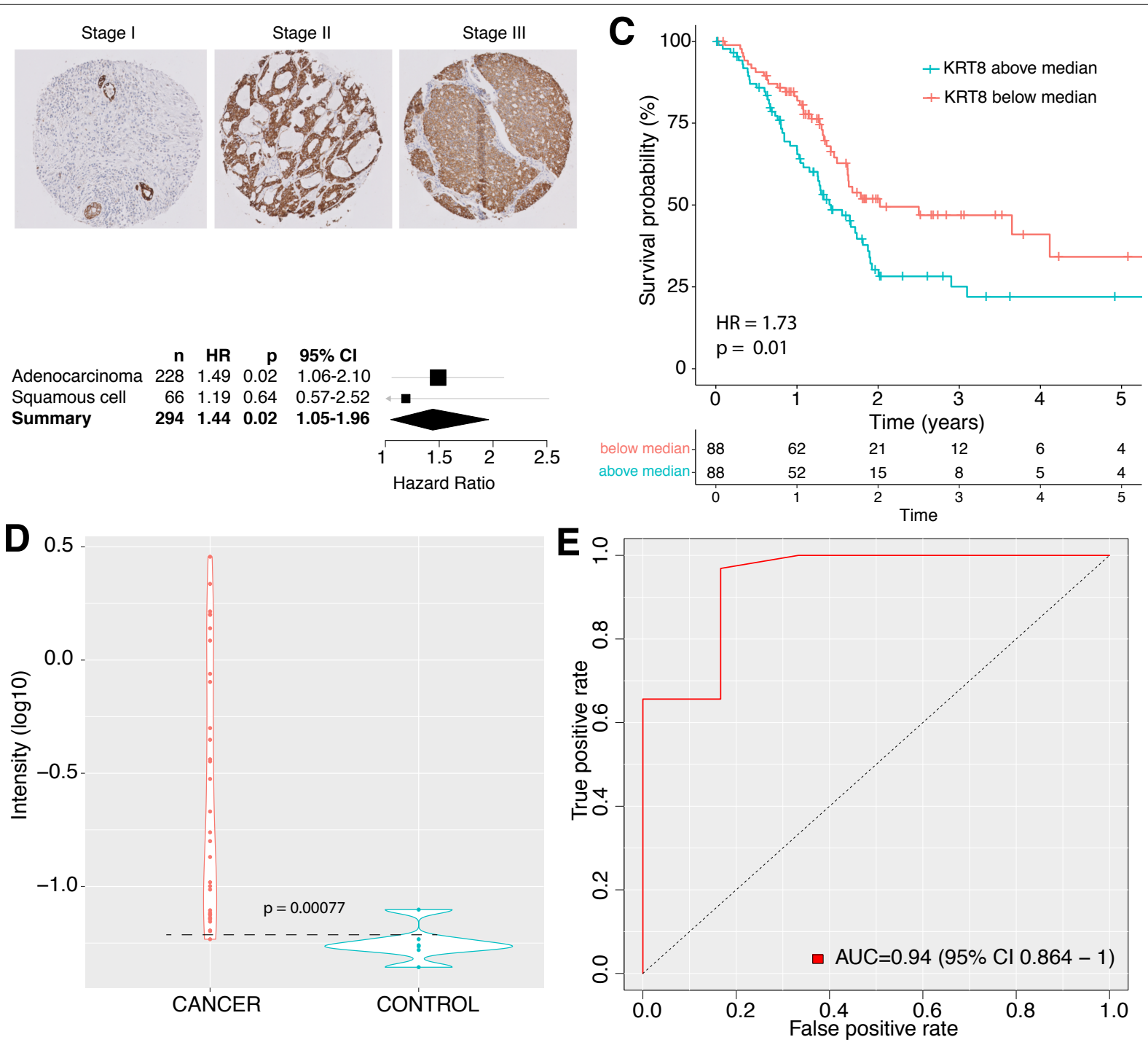

Figure 5. Protein measurement of KRT8 in cancer. A. IHC of lung adenocarcinoma TMAs for KRT8. B. Cox proportional hazard of 294 lung cancer samples stratified by KRT8 concentration. C. Survival of 176 patients with pancreatic cancer stratified by KRT8 expression relative to the median of the cohort. D-E. Violin (D) and ROC (E) plots of serum KRT8 as measured by ELISA in patients with pancreatic cancer and healthy controls. Dashed line represents the ELISA detection threshold. Width of a violin plot indicates density of samples, where each dot represents a sample.

\section{Protein expression of KRT8 is associated with poor outcomes in patients with lung adenocarcinoma}

Given robust hypomethylation of KRT8 across 9,855 samples from 23 cancers, over-expression across 6,781 biopsies from 5 cancers, strong association with chemo-resistance, and sustained correlation with p53-regulated genes both at single-cell and sample levels, we investigated whether KRT8 is also expressed at protein-level in tumor biopsies, and whether it is associated with survival in patients with either lung adenocarcinoma or lung squamous cell carcinoma. We stained tissue microarrays (TMAs) containing 294 lung tumors (228 lung adenocarcinoma, 66 lung squamous cell carcinoma) resected from patients at Stanford Hospital for KRT8 protein (Supplementary Table 3). An expert pathologist (MO) rated the maximum intensity of cancerous cell KRT8 staining in each TMA (Figure 5A). Out of the 294 samples, $5(1.7 \%)$ scored as $1+, 35(11.9 \%)$ as 1-2+, $55(18.7 \%)$ as $1-3+, 8(2.72 \%)$ as $2+, 85(28.9 \%)$ as $2-3+$ and $106(36.1 \%)$ as $3+$. In a multivariable cox regression model, KRT8 intensity was a significant predictor of mortality after adjusting for sex and age at diagnosis in lung 
medRxiv preprint doi: https://doi.org/10.1101/2020.10.01.20205450; this version posted October 4, 2020. The copyright holder for this preprint (which was not certified by peer review) is the author/funder, who has granted medRxiv a license to display the preprint in perpetuity. It is made available under a CC-BY-NC-ND 4.0 International license .

adenocarcinoma (Hazard Ratio $=1.49,95 \% \mathrm{CI}=1.06-2.10, \mathrm{p}=0.02)$, but not in squamous cell $($ Hazard Ratio $=$ $1.19,95 \% \mathrm{CI}=0.57-2.52, \mathrm{p}=0.65$; Figure $5 \mathrm{~B})$.

\section{Higher RNA expression of KRT8 is associated with reduced survival in patients with pancreatic cancer}

Next, we investigated whether KRT8 tumor gene expression is a prognostic marker of survival. We downloaded KRT8 expression and corresponding survival data for 176 patients with stage I-IV pancreatic cancer from Human Protein Atlas (Supplementary Table 4). We classified patients as either "High KRT8" or "Low KRT8" if their KRT8 expression was above or below the median KRT8 expression of the cohort (363.5 FPKM), respectively. Patients in the "High KRT8" group had an increased risk of mortality (cox proportional hazard ratio $=1.73 \mathrm{p}=$ 0.01 Figure 5C).

\section{Serum KRT8 discriminates between healthy and pancreatic patients and correlates with survival time}

Finally, we explored the potential of KRT8 as a minimally invasive biomarker. We measured KRT8 concentration in serum of 32 biopsy-confirmed patients with pancreatic ductal adenocarcinoma and six healthy controls by enzyme-linked immunosorbent assays (ELISA). Samples were collected from Stanford Hospital (Supplementary Table 5). The mean KRT8 concentration was significantly higher in the pancreatic cancer patients compared to that of healthy controls $(p=7.7 e-4$; Figure 5D). Samples were considered KRT8+ if they had a measured KRT8 value about the detectability limit of the ELISA (0.06 RLU). KRT8+ status distinguished patients with pancreatic cancer from healthy controls with an area under the curve (AUC) of 0.94 (Figure 5E) and an area under the precision recall curve (AUPRC) of 0.99 (Supplementary Figure 4).

\section{Discussion}

Only a fraction of molecular cancer biomarkers published in academic literature are reproducible in follow-up studies. The first step to identifying a robust biomarker is to ensure that the discovery phase has included a heterogeneous set of samples, platforms, and measurement technologies. Here, we identified KRT8 as such a biomarker by integrating DNA methylation profiling of 2019 samples across 10 cancers from the TCGA. We then validated that KRT8 is a robust biomarker on 7836 samples in 21 cancers measured with a different DNA methylation platform within the TCGA. We next analyzed the diagnostic and prognostic value of tumor KRT8 gene and protein expression as well as serum KRT8 using ELISA in over 7000 samples spanning 10 years, multiple platforms, and data repositories.

Pan-cancer methylation findings have been hindered by questions about batch effects and platform bias ${ }^{39}$. In this work, we used samples run on Illumina 27 platform as our discovery data and Illumina 450 as validation. KRT8 was significantly hypomethlyated in both platforms, suggesting it is robust to platform bias. While TCGA has gene expression data, we chose to use microarray samples from the NCBI GEO to ensure that our findings would be robust to data type, batch effect, and platform.

Single cell analysis has broadened our understanding of tumor heterogeneity, but it can be difficult to interpret the immediate translational value of a single time point scRNA-seq analysis. Here, we show that intra-cellular gene-gene correlations can suggest overlooked gene functions. Additionally, by replicating the correlations found at the single cell level in bulk tissue microarrays, we propose a strategy for validating expression patterns seen in the single cell level.

Our study has several limitations. First, it does not include the entirety of all cancer data available in the public sphere, and thus presents an incomplete picture of KRT8 across all data. However, this study used 17149 samples across 23 cancers, which still includes significant amount of biological, clinical, and technical heterogeneity in the real world patient population. Further, we have previously shown that 4-5 independent datasets with a total of approximately 200-250 samples substantially increases the probability of validation in independent cohrots ${ }^{23}$. Second, we only required two control samples in the methylation discovery analysis, which could have led to false positive or patient-specific effects within a datasets. However, the integration of all the discovery cohorts 
and independent validation using Illumina 450 methylation platform substantially mitigated the effect of a single cancer outlier. In addition, our rigorous downstream analysis of gene expression from 6781 samples in 57 datasets from 5 cancers provide strong evidence of the robustness of our analyses. Third, we chose only the top gene and validated it here. It is possible that other genes may provide equal or greater prognostic value than KRT8. However, our aim is to demonstrate the value of the framework we propose here and thus we explored only the most promising gene, KRT8. Forth, we do not provide any indication of the mechanism underlying the prognostic value of KRT8. It may be as straightforward as increasing epithelial cancer cell numbers results in more KRT8 released into the bloodstream, or perhaps there is a more complex biological phenomenon at work. These questions can only be answered with follow-up hypothesis-driven research.

Previous reports have identified role of KRT8 in the progression of lung and renal cancer. ${ }^{40,41}$ However, KRT8 has never been shown to be overrepresented across cancers in a multi-omic analysis. One GEO dataset (GSE15932) contained expression from peripheral blood samples. In this dataset, KRT8 expression distinguished cancerous from healthy patients, suggesting that circulating KRT8 RNA may be a candidate for a diagnostic blood biomarker. Biomarkers not only have diagnostic and prognostic implications, but are also helpful for measurement of treatment responses, surveillance for tumor recurrence and guiding clinical decisions. For many cancers, there is not a single blood biomarker; others like pancreatic cancer have one or two unreliable screening biomarkers. CA19-9 is used as a biomarker in pancreatic cancer, but due to its limitations and the low prevalence of pancreatic cancer is only used to monitor for reoccurrence. ${ }^{42}$ Here we show the potential use of serum KRT8 protein as a blood biomarker in pancreatic cancer. Given that we identified KRT8 as overexpressed across cancers, it stands to reason that KRT8 may be useful as a peripheral biomarker in other cancers as well.

Most importantly, this work demonstrates a strategy to translate large molecular analyses into specific, clinically relevant hypotheses. Omics sciences enable complex biological systems to be visualized in a holistic and integrative manner. Application of systems biology to interpret large multidimensional omics data across cancer types will enable the robust identification of biomarkers that share common pathophysiology, which can potentially be further explored for pan-cancer interventions

\section{Ethics approval and consent to participate}

All aspects of this study were approved by the Stanford Institutional Review Board in accordance with the Declaration of Helsinki guidelines for the ethical conduct of research. The reference number for the approval is IRB-20170. A waiver of informed consent was obtained for the subjects in this study according to Stanford's Institutional Review Board policy since this was a retrospective study of both alive and deceased patients, many of whom were lost to follow-up.

\section{Availability of data and materials}

Microarray data are available from the NCBI GEO at: https://www.ncbi.nlm.nih.gov/geo/ The accession numbers and corresponding links for the individual studies are listed in Supplemental Table 6.

\section{Acknowledgements}

We would like to acknowledge Julien Sage for his constructive feedback throughout this project.

\section{References}

1 Goossens, N., Nakagawa, S., Sun, X. \& Hoshida, Y. Cancer biomarker discovery and validation. Transl Cancer Res 4, 256-269, doi:10.3978/j.issn.2218-676X.2015.06.04 (2015).

2 Hiom, S. C. Diagnosing cancer earlier: reviewing the evidence for improving cancer survival. Br J Cancer 112 Suppl 1, S1-5, doi:10.1038/bjc.2015.23 (2015).

3 de Groot, P. M., Wu, C. C., Carter, B. W. \& Munden, R. F. The epidemiology of lung cancer. Transl Lung Cancer Res 7, 220-233, doi:10.21037/tlcr.2018.05.06 (2018).

4 Lemjabbar-Alaoui, H., Hassan, O. U., Yang, Y. W. \& Buchanan, P. Lung cancer: Biology and treatment options. Biochim Biophys Acta 1856, 189-210, doi:10.1016/j.bbcan.2015.08.002 (2015). 
medRxiv preprint doi: https://doi.org/10.1101/2020.10.01.20205450; this version posted October 4, 2020. The copyright holder for this preprint (which was not certified by peer review) is the author/funder, who has granted medRxiv a license to display the preprint in perpetuity. It is made available under a CC-BY-NC-ND 4.0 International license .

Uramoto, H. \& Tanaka, F. Recurrence after surgery in patients with NSCLC. Transl Lung Cancer Res 3, 242-249, doi:10.3978/j.issn.2218-6751.2013.12.05 (2014).

Tomczak, K., Czerwińska, P. \& Wiznerowicz, M. The Cancer Genome Atlas (TCGA): an immeasurable source of knowledge. Contemp Oncol (Pozn) 19, A68-77, doi:10.5114/wo.2014.47136 (2015).

Tang, H. et al. Comprehensive evaluation of published gene expression prognostic signatures for biomarker-based lung cancer clinical studies. Ann Oncol 28, 733-740, doi:10.1093/annonc/mdw683 (2017). Insights 12, 1177271917715236, doi:10.1177/1177271917715236 (2017). Wang, E., Cho, W. C. S., Wong, S. C. C. \& Liu, S. Disease Biomarkers for Precision Medicine: Challenges and Future Opportunities. Genomics Proteomics Bioinformatics 15, 57-58, doi:10.1016/j.gpb.2017.04.001 (2017). Drucker, E. \& Krapfenbauer, K. Pitfalls and limitations in translation from biomarker discovery to clinical utility in predictive and personalised medicine. EPMA J4, 7, doi:10.1186/1878-5085-4-7 (2013).

1 Leygo, C. et al. DNA Methylation as a Noninvasive Epigenetic Biomarker for the Detection of Cancer. Dis Markers 2017, 3726595, doi:10.1155/2017/3726595 (2017).

12 Paziewska, A. et al. DNA methylation status is more reliable than gene expression at detecting cancer in prostate biopsy. Br J Cancer 111, 781-789, doi:10.1038/bjc.2014.337 (2014).

13 Yang, X., Gao, L. \& Zhang, S. Comparative pan-cancer DNA methylation analysis reveals cancer common and specific patterns. Brief Bioinform 18, 761-773, doi:10.1093/bib/bbw063 (2017).

14 Dong, L. \& Ren, H. Blood-based DNA Methylation Biomarkers for Early Detection of Colorectal Cancer. J Proteomics Bioinform 11, 120-126, doi:10.4172/jpb.1000477 (2018).

15 Belinsky, S. A. et al. Gene Methylation Biomarkers in Sputum and Plasma as Predictors for Lung Cancer Recurrence. Cancer Prev Res (Phila) 10, 635-640, doi:10.1158/1940-6207.CAPR-17-0177 (2017).

16 Kisiel, J. B. et al. New DNA Methylation Markers for Pancreatic Cancer: Discovery, Tissue Validation, and Pilot Testing in Pancreatic Juice. Clin Cancer Res 21, 4473-4481, doi:10.1158/1078-0432.CCR-142469 (2015).

17 Mikeska, T. \& Craig, J. M. DNA methylation biomarkers: cancer and beyond. Genes (Basel) 5, 821-864, doi:10.3390/genes5030821 (2014).

18 Jain, S., Wojdacz, T. K. \& Su, Y. H. Challenges for the application of DNA methylation biomarkers in molecular diagnostic testing for cancer. Expert Rev Mol Diagn 13, 283-294, doi:10.1586/erm.13.9 (2013).

19 Weinstein, J. N. et al. The Cancer Genome Atlas Pan-Cancer analysis project. Nat Genet 45, 1113-1120, doi:10.1038/ng.2764 (2013).

20 Clough, E. \& Barrett, T. The Gene Expression Omnibus Database. Methods Mol Biol 1418, 93-110, doi:10.1007/978-1-4939-3578-9_5 (2016).

21 Scott, M. K. D. et al. Increased monocyte count as a cellular biomarker for poor outcomes in fibrotic diseases: a retrospective, multicentre cohort study. Lancet Respir Med 7, 497-508, doi:10.1016/S22132600(18)30508-3 (2019).

22 Sweeney, T. E., Braviak, L., Tato, C. M. \& Khatri, P. Genome-wide expression for diagnosis of pulmonary tuberculosis: a multicohort analysis. Lancet Respir Med 4, 213-224, doi:10.1016/S2213-2600(16)000485 (2016).

23 Sweeney, T. E., Haynes, W. A., Vallania, F., Ioannidis, J. P. \& Khatri, P. Methods to increase reproducibility in differential gene expression via meta-analysis. Nucleic Acids Res 45, e1, doi:10.1093/nar/gkw797 (2017).

24 Benjamini, Y., Drai, D., Elmer, G., Kafkafi, N. \& Golani, I. Controlling the false discovery rate in behavior genetics research. Behav Brain Res 125, 279-284, doi:10.1016/s0166-4328(01)00297-2 (2001).

25 Lambrechts, D. et al. Phenotype molding of stromal cells in the lung tumor microenvironment. Nat Med 24, 1277-1289, doi:10.1038/s41591-018-0096-5 (2018).

26 Uhlen, M. et al. A pathology atlas of the human cancer transcriptome. Science 357, doi:10.1126/science.aan2507 (2017). 
medRxiv preprint doi: https://doi.org/10.1101/2020.10.01.20205450; this version posted October 4, 2020. The copyright holder for this preprint (which was not certified by peer review) is the author/funder, who has granted medRxiv a license to display the preprint in perpetuity. It is made available under a CC-BY-NC-ND 4.0 International license .

Nair, V. S., Gevaert, O., Davidzon, G., Plevritis, S. K. \& West, R. NF-кB protein expression associates with (18)F-FDG PET tumor uptake in non-small cell lung cancer: a radiogenomics validation study to understand tumor metabolism. Lung Cancer 83, 189-196, doi:10.1016/j.lungcan.2013.11.001 (2014). Neesse, A., Griesmann, H., Gress, T. M. \& Michl, P. Claudin-4 as therapeutic target in cancer. Arch Biochem Biophys 524, 64-70, doi:10.1016/j.abb.2012.01.009 (2012).

29 Losi-Guembarovski, R., Kuasne, H., Guembarovski, A. L., Rainho, C. A. \& Cólus, I. M. DNA methylation patterns of the CDH1, RARB, and SFN genes in choroid plexus tumors. Cancer Genet Cytogenet 179, 140-145, doi:10.1016/j.cancergencyto.2007.05.029 (2007).

30 Chen, Y. et al. PAX1 and SOX1 methylation as an initial screening method for cervical cancer: a metaanalysis of individual studies in Asians. Ann Transl Med 4, 365, doi:10.21037/atm.2016.09.30 (2016).

31 Okada, T. et al. TWIST1 hypermethylation is observed frequently in colorectal tumors and its overexpression is associated with unfavorable outcomes in patients with colorectal cancer. Genes Chromosomes Cancer 49, 452-462, doi:10.1002/gcc.20755 (2010).

32 Hellebrekers, D. M. et al. GATA4 and GATA5 are potential tumor suppressors and biomarkers in colorectal cancer. Clin Cancer Res 15, 3990-3997, doi:10.1158/1078-0432.CCR-09-0055 (2009).

33 Spainhour, J. C., Lim, H. S., Yi, S. V. \& Qiu, P. Correlation Patterns Between DNA Methylation and Gene Expression in The Cancer Genome Atlas. Cancer Inform 18, 1176935119828776, doi:10.1177/1176935119828776 (2019).

34 Eisenberg, E. \& Levanon, E. Y. Human housekeeping genes, revisited. Trends Genet 29, 569-574, doi:10.1016/j.tig.2013.05.010 (2013).

35 Silver, N., Best, S., Jiang, J. \& Thein, S. L. Selection of housekeeping genes for gene expression studies in human reticulocytes using real-time PCR. BMC Mol Biol 7, 33, doi:10.1186/1471-2199-7-33 (2006).

36 Schultz, M. D. et al. Human body epigenome maps reveal noncanonical DNA methylation variation. Nature 523, 212-216, doi:10.1038/nature14465 (2015).

37 Fabregat, A. et al. The Reactome pathway Knowledgebase. Nucleic Acids Res 44, D481-487, doi:10.1093/nar/gkv1351 (2016).

38 Lo, H. W. et al. Identification and functional characterization of the human glutathione S-transferase P1 gene as a novel transcriptional target of the p53 tumor suppressor gene. Mol Cancer Res 6, 843-850, doi:10.1158/1541-7786.MCR-07-2105 (2008).

39 Witte, T., Plass, C. \& Gerhauser, C. Pan-cancer patterns of DNA methylation. Genome Med 6, 66, doi:10.1186/s13073-014-0066-6 (2014).

40 Tan, H. S. et al. KRT8 upregulation promotes tumor metastasis and is predictive of a poor prognosis in clear cell renal cell carcinoma. Oncotarget 8, 76189-76203, doi:10.18632/oncotarget.19198 (2017).

41 Wang, W., He, J., Lu, H., Kong, Q. \& Lin, S. KRT8 and KRT19, associated with EMT, are hypomethylated and overexpressed in lung adenocarcinoma and link to unfavorable prognosis. Biosci Rep 40, doi:10.1042/BSR20193468 (2020).

42 Scarà, S., Bottoni, P. \& Scatena, R. CA 19-9: Biochemical and Clinical Aspects. Adv Exp Med Biol 867, 247-260, doi:10.1007/978-94-017-7215-0_15 (2015). 